\title{
Phase I study of high-dose ascorbic acid with mFOLFOX6 or FOLFIRI in patients with metastatic colorectal cancer or gastric cancer
}

Feng Wang ${ }^{1,2+}$, Ming-Ming He ${ }^{1,2+}$, Zi-Xian Wang ${ }^{1,2+}$, Su Li ${ }^{1,3}$, Ying Jin ${ }^{1,2}$, Chao Ren ${ }^{1,2}$, Si-Mei Shi ${ }^{1,2}$, Bing-Tian Bi ${ }^{1,3}$, Shuang-Zhen Chen ${ }^{1,2}$, Zhi-Da Lv',2, Jia-Jia Hu' ${ }^{1}$, Zhi-Qiang Wang ${ }^{1,2}$, Feng-Hua Wang ${ }^{1,2}$, De-Shen Wang ${ }^{1,2}$, Yu-Hong Li ${ }^{1,2}$ and Rui-Hua Xu ${ }^{1,2^{*}}$

\begin{abstract}
Background: Preclinical studies suggest synergistic effectiveness of ascorbic acid (AA, vitamin C) and cytotoxic agents in gastrointestinal malignancies. This phase 1 study aimed to establish the maximum tolerated dose (MTD) and recommended phase 2 dose (RP2D) of AA combined with mFOLFOX6 or FOLFIRI regimens in patients with metastatic colorectal cancer ( $m C R C$ ) or gastric cancer (mGC).

Methods: In the dose-escalation phase, patients received AA (0.2-1.5 g/ $/ \mathrm{kg}$, 3-h infusion, once daily, days 1-3) with mFOLFOX6 or FOLFIRI in a 14-day cycle until the MTD was reached. In the speed-expansion phase, AA was administered at the MTD or at $1.5 \mathrm{~g} / \mathrm{kg}$ if the MTD was not reached at a fixed rate of $0.6,0.8$ or $1 \mathrm{~g} / \mathrm{min}$. Pharmacokinetics and preliminary efficacy were also assessed.

Results: Thirty-six patients were enrolled. The MTD was not reached. The RP2D was established as AA at $1.5 \mathrm{~g} / \mathrm{kg} /$ day, days 1-3, with mFOLFOX6 or FOLFIRI. No dose-limiting toxicity (DLT) was detected during dose escalation. The most common treatment-emergent adverse events (TRAEs) were sensory neuropathy (50\%), nausea (38.9\%), vomiting (36.1\%) and neutropenia (27.8\%). Grade 3-4 TRAEs were neutropenia (13.9\%), sensory neuropathy (2.8\%), vomiting (2.8\%), diarrhea (2.8\%) and leukopenia (2.8\%). AA exposure was dose-proportional. The objective response rate was 58.3\%, and the disease control rate was 95.8\%. No difference in efficacy was found between mCRC patients with wild-type RAS/BRAF and mutant RAS or BRAF.
\end{abstract}

Conclusions: The favorable safety profile and preliminary efficacy of AA plus mFOLFOX6/FOLFIRI support further evaluation of this combination in $\mathrm{mCRC}$ or $\mathrm{mGC}$.

Trial registration: ClinicalTrial.gov Identifier: NCT02969681.

Keywords: Ascorbic acid, Metastatic colorectal cancer, Metastatic gastric cancer, Recommended phase 2 dose; chemotherapy

\footnotetext{
* Correspondence: xurh@sysucc.org.cn

†Feng Wang, Ming-Ming He and Zi-Xian Wang contributed equally to this

work.

${ }^{1}$ State Key Laboratory of Oncology in South China, Collaborative Innovation

Center for Cancer Medicine, Sun Yat-sen University Cancer Center,

Guangzhou 510060, People's Republic of China

Department of Medical Oncology, Sun Yat-sen University Cancer Center,

Guangzhou 510060, China

Full list of author information is available at the end of the article
}

(c) The Author(s). 2019 Open Access This article is distributed under the terms of the Creative Commons Attribution 4.0 International License (http://creativecommons.org/licenses/by/4.0/), which permits unrestricted use, distribution, and reproduction in any medium, provided you give appropriate credit to the original author(s) and the source, provide a link to the Creative Commons license, and indicate if changes were made. The Creative Commons Public Domain Dedication waiver (http://creativecommons.org/publicdomain/zero/1.0/) applies to the data made available in this article, unless otherwise stated. 


\section{Background}

The role of ascorbic acid (AA, vitamin $\mathrm{C}$ ) in both cancer prevention and treatment has been controversial [1]. Epidemiological evidence suggests that ingestion of AA-rich foods might be associated with reduced cancer incidence [2]. However, this was not confirmed in randomized intervention trials [3, 4]. In the 1970s, a retrospective study by Ewan Cameron and Linus Pauling reported that high doses of intravenous and oral AA increased the average survival of advanced cancer patients compared with that of controls [5, 6]. However, two subsequent placebo-controlled randomized clinical trials investigating the same dose of oral $\mathrm{AA}$ in patients with advanced cancer were both negative, leading to decreased interest in the use of AA in cancer treatment $[7,8]$.

Recent preclinical and clinical studies have regenerated interest in the potential anticancer effects of AA [9-11]. Preclinical studies have reported that human colorectal cancer cells harboring KRAS or BRAF mutations are selectively killed when exposed to high levels of AA [12]. Treatment with AA also suppresses colony formation and leukemia progression in primary human leukemia patient-derived xenografts (PDXs) [13]. Clinical studies have shown that high (millimolar) plasma AA concentrations, which are selectively cytotoxic to many neoplastic cell lines, can only be achieved with intravenous infusion rather than oral administration [14-16].

Although the high AA monotherapy i.v. dose was well-tolerated, it failed to demonstrate anticancer activity in patients with previously treated advanced malignancies [17]. However, preclinical studies have suggested a synergistic effect between cytotoxic agents and AA, in which high concentrations of this redox-active compound might modify either the treatment response or toxicity [11, 17]. Moreover, existing evidence suggests that high-dose AA can safely be given alongside cytotoxic agents, such as gemcitabine, paclitaxel, and carboplatin $[18,19]$.

The present phase I study (ClinicalTrial.gov Identifier: NCT02969681) aimed to assess the safety, pharmacokinetic (PK) profile, and preliminary efficacy of AA in combination with mFOLFOX6 or FOLFIRI regimens in Chinese patients with metastatic colorectal cancer (mCRC) or gastric cancer (mGC).

\section{Methods}

\section{Study design}

This phase 1 open-label, single-center, dose-escalation, and speed-expansion study evaluated AA in combination with mFOLFOX6 or FOLFIRI in patients with $\mathrm{mCRC}$ or mGC. The primary objective was to evaluate the safety profile and determine the maximum tolerated dose (MTD) and the recommended phase 2 dose (RP2D) of AA when coadministered with mFOLFOX6 or FOLFIRI.
Secondary objectives were to assess the PK profile and preliminary anti-tumor activity of AA in combination with mFOLFOX6 or FOLFIRI. Patients received AA and mFOLFOX6 or FOLFIRI in 14-daycycles. AA was administered on days 1-3 with chemotherapy (Fig. 1). The trial was registered with the ClinicalTrials.gov registry on November 21, 2016 and was approved by the Independent Institute Research Ethics Committee at the Sun Yat-sen University Cancer Center prior to initiation. This trial was conducted in accordance with the Declaration of Helsinki, the guidelines for Good Clinical Practice, the European Union Clinical Trial Directive, and local regulations. All participants provided written informed consent. This report adheres to the CONSORT guidelines.

\section{Patients}

Patients with histologically confirmed mCRC or mGC who were scheduled to receive mFOLFOX6 or FOLFIRI as first-line or second-line chemotherapy regimens without a targeted therapy agent were enrolled in part 1 . Patients with histologically confirmed mCRC who were scheduled to receive mFOLFOX6 with or without bevacizumab as the first-line therapy were enrolled in part 2 . In addition, patients were required to be $\geq 18$ years and $\leq$ 75 years of age; have an Eastern Cooperative Oncology Group (ECOG) performance status of $0-1$; a G6PD status greater than the lower limit of normal; a life expectancy of at least 12 weeks; and adequate hematologic function $(A N C \geq 1500 / \mathrm{mm} 3$; hemoglobin $>8 \mathrm{~g} / \mathrm{dL}$; platelet $\geq 100,000 / \mathrm{mm} 3$ ), renal function [creatinine $\leq 1.5 \times$ upper limit of normal (ULN); if the creatinine level was elevated but $\leq 1.5 \times$ the ULN, a 24 -h creatinine clearance would be obtained, and creatinine clearance was required to be $\geq 50 \mathrm{ml} / \mathrm{min}$ (calculated according to Cockroft and Gault)] and hepatic function [transaminase $(\mathrm{AST} / \mathrm{ALT}) \leq 2.5 \times \mathrm{ULN}$ and bilirubin levels $\leq 1.5 \times \mathrm{ULN}$ without liver metastasis; transaminase (AST/ALT) $\leq 5 \times$ ULN and bilirubin levels $\leq 1.5 \times$ ULN with liver metastasis]. Women of childbearing potential needed to confirm a negative pregnancy test and practice effective contraception during the study. Written informed consent was required.

The exclusion criteria were failure of both oxaliplatinand irinotecan-based regimens; surgery (excluding diagnostic biopsy) or irradiation within 3 weeks prior to study entry; administration of any investigational drug or agent/procedure, i.e., participation in another trial within 4 weeks before beginning treatment; concurrent chronic systemic immune therapy, chemotherapy, radiation therapy (palliative radiation therapy allowed) or hormone therapy not indicated in the study protocol; brain metastasis (known or suspected); pregnant or lactating women; other uncontrolled concomitant illness, including serious uncontrolled intercurrent infection; 


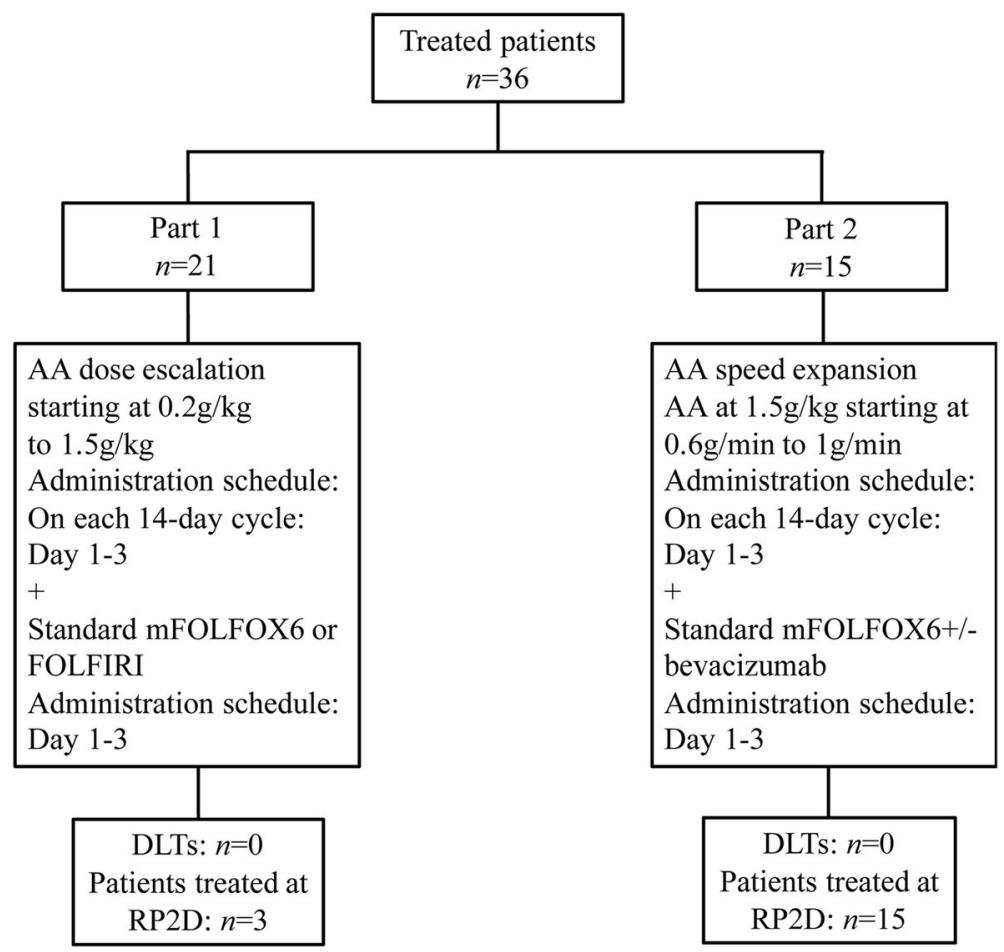

Fig. 1 Study design. Ascorbic acid was administered in both study parts with 5-FU continuous infusion. Standard mFOLOX6 comprised a standard dose of $85 \mathrm{mg} / \mathrm{m}^{2}$ oxaliplatin on day $1+$ leucovorin at $400 \mathrm{mg} / \mathrm{m}^{2}$ (2-h infusion during oxaliplatin administration) $+5 \mathrm{FU}$ at $400 \mathrm{mg} / \mathrm{m}^{2}$ (bolus immediately following irinotecan administration) and $2400 \mathrm{mg} / \mathrm{m}^{2}$ (46-h continuous infusion), every 2 weeks. Standard FOLFIRI consisted of a standard dose of irinotecan at $180 \mathrm{mg} / \mathrm{m} 2$ (90-min infusion) + leucovorin $400 \mathrm{mg} / \mathrm{m}^{2}$ (2-h infusion during irinotecan administration) + 5-FU at $400 \mathrm{mg} / \mathrm{m} 2$ (bolus immediately following irinotecan administration) and $2400 \mathrm{mg} / \mathrm{m}^{2}$ ( $46-\mathrm{h}$ continuous infusion), every 2 weeks. In part 1 , AA was administered once daily at $180 \mathrm{~min}$ (part 1) or at a fixed rate of $0.6,0.8$ and $1 \mathrm{~g} / \mathrm{min}$ (part 2) for three consecutive days with continuous 5 -FU infusion in a 14-day cycle

known allergy or any other adverse reaction to any of the drugs or to any related compound; previous (within 5 years) or concurrent malignancies at other sites with the exception of surgically cured or adequately treated carcinoma in situ of the cervix and basal cell carcinoma of the skin; patients who were on strong inducers of CYP3A4, including but not limited to aminoglutethimide, bexarotene, bosentan, carbamazepine, dexamethasone, efavirenz, fosphenytoin, griseofulvin, modafinil, nafcillin, nevirapine, oxcarbazepine, phenobarbital, phenytoin, primidone, rifabutin, rifampin, rifapentine, and St. John's wort; a medical, social or psychological condition, which in the opinion of the investigator would not permit the patient to complete the study or sign a meaningful informed consent; organ allograft requiring immunosuppressive therapy; and patients with HIV infection.

\section{Treatment schedule}

In the dose-escalation phase (part 1), the starting dose of AA (3-h infusion, once daily) was $0.2 \mathrm{~g} / \mathrm{kg}$. Dose escalation of AA was performed according to the standard 3 +3 dose-finding scheme, in which up to three additional patients were enrolled at any dose level where a dose-limiting toxicity (DLT) was recorded. Subsequent doses were $0.4 \mathrm{~g} / \mathrm{kg}, 0.6 \mathrm{~g} / \mathrm{kg}, 0.8 \mathrm{~g} / \mathrm{kg}, 1.0 \mathrm{~g} / \mathrm{kg}, 1.2 \mathrm{~g} / \mathrm{kg}$ and $1.5 \mathrm{~g} / \mathrm{kg}$ until the MTD was reached. The MTD or RP2D were defined as the dose levels at which fewer than $33 \%$ of the enrolled patients experienced DLT. DLTs were defined as any grade 3 or 4 hematologic toxicity lasting 1 week or longer or as any grade 3 or 4 nonhematologic toxicity upon completion of one 2-week treatment cycle (14 days). Manageable nausea and vomiting, fatigue, anorexia, anemia, alopecia, alkaline phosphatase changes, fever without neutropenia, and local reactions were not included in the determination of DLT. Patients not completing the first cycle for reasons other than DLTs were considered not evaluable and replaced.

The chemotherapy backbone (mFOLFOX6 or FOLFIRI) was chosen at the investigators' discretion. The chemotherapy regimens were as follows: mFOLOX6 comprised oxaliplatin $85 \mathrm{mg} / \mathrm{m}^{2}$ on day 1 concurrent with leucovorin $400 \mathrm{mg} / \mathrm{m}^{2}$, a bolus of $5 \mathrm{FU} 400 \mathrm{mg} / \mathrm{m}^{2}$, followed by infusion of $5 \mathrm{FU}$ at $2400 \mathrm{mg} / \mathrm{m}^{2}$ over $46 \mathrm{~h}$, every 2 weeks; FOLFIRI comprised irinotecan CPT- $11180 \mathrm{mg} / \mathrm{m}^{2}$ on day 1 
concurrent with leucovorin $400 \mathrm{mg} / \mathrm{m}^{2}$, a bolus of $5 \mathrm{FU}$ $400 \mathrm{mg} / \mathrm{m}^{2}$, followed by infusion of $5 \mathrm{FU} 2400 \mathrm{mg} / \mathrm{m}^{2}$ over $46 \mathrm{~h}$, every 2 weeks. For patients with mCRC who chose to receive bevacizumab, the dose was $5 \mathrm{mg} / \mathrm{kg}$ every 2 weeks. In part 1, AA was administered once daily for 180 min for three consecutive days with mFOLFOX6 or FOLFIRI in a 14-day cycle.

In the speed-expansion phase (part 2), AA was administered at the MTD or $1.5 \mathrm{~g} / \mathrm{kg}$, if the MTD was not reached, once daily at a fixed rate of $0.6,0.8$ and $1 \mathrm{~g} / \mathrm{min}$ (five patients each) for three consecutive days with mFOLFOX6 in a 14-day cycle.

The treatment continued until 12 cycles, disease progression, unmanageable toxic effects, or withdrawal of consent. For patients who suffered from greater than grade 2 peripheral neurotoxicity, oxaliplatin treatment was stopped.

\section{Safety, pharmacokinetic, and efficacy assessments}

All treated patients were assessed for safety, including adverse events, complete blood count, laboratory assessments, vital signs, electrocardiogram, and physical examination. Adverse events were defined and graded according to the NCI Common Terminology Criteria for Adverse Events (CTCAE, v4.03) and were recorded from enrollment until 3 weeks after the last dose. Pre- and postdose 5 -ml blood samples were collected at prespecified time points in cycle 1 during and after infusions. Symptoms or side-effects that were associated with rapid infusion of high-osmolarity solution or treatment-related adverse events that were possibly attributed to high-dose AA were defined as DLT. [17]

Blood samples were taken immediately before infusion, mid-infusion, at the end-point, and at 1, 3, 6, and $12 \mathrm{~h}$ after the end-point. Patient plasma samples were placed in individual tubes, protected from light, and then frozen at $-80^{\circ} \mathrm{C}$ until the assays were carried out. Plasma samples for PK analyses were obtained on the first and third day of the first week of dosing. AA was measured in 3-ml blood samples at the indicated times by LC-MS/ MS. Briefly, plasma samples were diluted 100-times with normal saline containing $0.05 \%$ EDTA. An aliquot $(100 \mu \mathrm{l})$ of the diluted plasma was extracted by adding $400 \mu \mathrm{l}$ of acetonitrile with an internal standard. Samples were then vortexed for $1 \mathrm{~min}$ and centrifuged at 12000 $\mathrm{rpm}$ for $10 \mathrm{~min}$ at $4{ }^{\circ} \mathrm{C}$. A $2-\mu \mathrm{l}$ sample was injected into the high-performance liquid chromatography system using a temperature-controlled autosampling device maintained at $4{ }^{\circ} \mathrm{C}$. Separation of analytes was achieved using a PLRP-S column (Agilent, $150 \times 2.1 \mathrm{~mm}, 8 \mu \mathrm{m}$, $1000 \AA$ ). The mobile phase used for chromatographic separation consisted of water containing $0.1 \%$ formic acid as eluent $\mathrm{A}$ and acetonitrile as eluent B. The analytes were separated in a gradient at a flow rate of 0.4 $\mathrm{ml} / \mathrm{min}$. The column effluent was monitored using an AB Sciex 5500 system. The samples were analyzed using an electrospray probe in the negative ionization mode operating at the $\mathrm{m} / \mathrm{z}$ for $\mathrm{AA}$ and that of the internal standard $\mathrm{m} /$ $\mathrm{z}$ with a spray voltage of $-4500 \mathrm{~V}$. A good linearity was demonstrated within the range of $5-50 \mu \mathrm{g} / \mathrm{mL}$. The PK parameters were determined by WinNonlin software, including the maximal $\mathrm{mM}$ drug-concentration in plasma (Cmax), the area under the drug-concentration curve (AUC) from infusion start to extrapolated infinite time, clearance, and noncompartmental terminal elimination rate.

Computed tomography or MRI were performed within 4 weeks before treatment initiation, after every 3 cycles, and 3 weeks after the final dose. Tumor response was assessed using Response Evaluation Criteria in Solid Tumors (RECIST) v. 1.1.

\section{Statistical analyses}

Descriptive statistics were used for analysis of the safety, pharmacokinetics, and tumor response data. The study population for safety included patients who received at least one dose of the study medication. The tumor response was assessed in all patients who received at least one dose of the study medication, had measurable disease at the baseline per RECIST v1.1 as assessed by central review, and had at least one post-baseline scan. Progression-free survival (PFS) was measured from the date of first exposure to the study drugs to the earliest date of disease progression or death from any cause, whichever came first.

\section{Results}

\section{Patient characteristics and treatment exposure}

A total of 36 patients were enrolled in this phase I trial between March 18, 2017 and June 14, 2017 (Fig. 1). The demographics and baseline characteristics of the study participants are presented in Table 1 . Thirty patients with $\mathrm{mCRC}$ and six with mGC were recruited. Among the 30 patients with mCRC, 26 patients were assayed for KRAS, NRAS and BRAF status. Among them, 10 patients carried KRAS mutations, 2 patients carried BRAF V600E mutation and 14 had wild-type RAS and BRAF. Thirty-five patients received FOLFOX6 (first-line for all) and one received FOLFIRI (second-line) as the chemotherapy backbone. Fourteen of 30 (46.7\%) patients with mCRC received bevacizumab with chemotherapy and AA.

Overall, patients were treated with a median of 8 cycles of chemotherapy with AA (range, 1-12). At the time of the data cutoff, all patients had discontinued AA and/or the study chemotherapeutic regimens. The reasons for discontinuation of AA and/or chemotherapy included completion of 12 cycles (5 patients, $13.9 \%$ ), a switch to capecitabine-based maintenance therapy (7 patients, $19.4 \%$ ), surgery (4 patients, $11.1 \%$ ), disease progression 
Table 1 Patient characteristics $(N=36)$

\begin{tabular}{|c|c|}
\hline Characteristics & No. (\%) \\
\hline \multicolumn{2}{|l|}{ Age, years } \\
\hline Median (range) & $53(27-75)$ \\
\hline$<65$ & $28(77.8)$ \\
\hline$\geq 65$ & $8(22.2)$ \\
\hline \multicolumn{2}{|l|}{ Sex } \\
\hline Male & $21(58.3)$ \\
\hline Female & $15(41.7)$ \\
\hline \multicolumn{2}{|l|}{ ECOG performance status } \\
\hline 0 & $2(5.6)$ \\
\hline 1 & $33(91.7)$ \\
\hline 2 & $1(2.8)$ \\
\hline Weight, kg, median (range) & $57.0(39.0-77.0)$ \\
\hline \multicolumn{2}{|l|}{ Cancer type } \\
\hline Colorectal & $30(83.3)$ \\
\hline Gastric & $6(16.7)$ \\
\hline \multicolumn{2}{|l|}{ Stage at diagnosis } \\
\hline Synchronous metastasis & $28(77.8)$ \\
\hline Metachronous metastasis & $8(22.2)$ \\
\hline \multicolumn{2}{|l|}{ Prior chemotherapy } \\
\hline Yes & $7(19.4)$ \\
\hline No & 29 (80.6) \\
\hline \multicolumn{2}{|c|}{ Previous surgery to the primary site } \\
\hline Yes & $19(52.8)$ \\
\hline No & $17(47.2)$ \\
\hline \multicolumn{2}{|l|}{ Add Avastin } \\
\hline Yes & $14(38.9)$ \\
\hline No & $22(61.1)$ \\
\hline \multicolumn{2}{|l|}{ RAS and BRAF status } \\
\hline KRAS mutant & $10(27.8)$ \\
\hline BRAF V600E mutant & $2(5.6)$ \\
\hline RAS and BRAF wild-type & $14(38.9)$ \\
\hline Unknown & $10(27.8)$ \\
\hline
\end{tabular}

ECOG Eastern Cooperative Oncology Group

(11 patients, 30.6\%), a loss of follow-up (5 patients, $13.9 \%)$, and abandonment of chemotherapy $(4,11.1 \%)$.

\section{MTD}

Twenty-one patients enrolled in the dose-escalation group were evaluable for DLT. No DLT was observed at any of the doses, and the MTD was not reached. Thus, the RP2D was established as AA at $1.5 \mathrm{~g} / \mathrm{kg}$ once daily for three consecutive days in combination with standard mFOLFOX6 with or without bevacizumab every 14 days. All 15 patients enrolled into the speed-escalation group were evaluated for DLT. No DLT was observed at any of the speeds.

\section{Safety}

All 36 patients enrolled were evaluated for safety. Treatment-related adverse events (TEAEs) of any grade were recorded in 32 patients (88.9\%) (Table 2). The most common all-grade TEAEs were peripheral sensory neuropathy $(50.0 \%)$, nausea (38.9\%), vomiting (36.1\%) and neutropenia (27.8\%). Grade 3 TEAEs occurred in nine (25.0\%) patients, five (13.9\%) of whom experienced grade 3 neutropenia, one (2.8\%) experienced vomiting, one (2.8\%) grade 3 diarrhea, one (2.8\%) leukopenia, and one (2.8\%) grade 3 peripheral sensory neuropathy. Grade 4 TEAEs occurred in one $(2.8 \%)$ patient who had neutropenia. No 5 TEAEs and no serious adverse events were detected. No patients discontinued AA and/or chemotherapy due to intolerable toxicity. Among the 26 patients treated with at least 6 cycles of oxaliplatin, grade 1 sensory neuropathy occurred in nine patients $(25.0 \%)$, grade 2 in eight patients $(22.2 \%)$, and grade 3 in one patient $(2.8 \%)$.

\section{Pharmacokinetics and pharmacodynamics}

During continuous infusion, the plasma AA concentrations rose from normal values $(<0.1 \mathrm{mmol} / \mathrm{L})$ to concentrations that peaked at the end of the infusion (Fig. 2). The AA was eliminated by simple first-order kinetics. The AA elimination half-life $\left(t_{1 / 2}\right)$, clearance, $C_{\max }$, and AUC in the fixed 3 -h infusion group are presented in Table 3. AA did not accumulate to any significant level during 3 consecutive daily administrations, and the $t_{1 / 2}$, $\mathrm{C}_{\text {max }}$, and $\mathrm{AUC}$ values of $\mathrm{AA}$ for each patient did not systematically change between the first and the third treatment. The $\mathrm{C}_{\max }$ and AUC values increased between $3.5 \sim 17.8 \mathrm{mmol} / \mathrm{L}$ and $14.9 \sim 89.5 \mathrm{mmol} / \mathrm{L} * \mathrm{~h}$, respectively. On day 1 and day 3 of administration, the correlation coefficients $\left(R^{2}\right)$ were 0.9523 and 0.9195 for the $C_{\max }$ versus dose, and 0.9697 and 0.8657 for the AUC versus dose in the dosing range (Additional file 1: Figure S1). The $t_{1 / 2}$ values $(1.4 \sim 3.1 \mathrm{~h})$ of AA were similar for all patients in all cohorts. However, the $\mathrm{C}_{\max }$ and AUC appeared to reach maximum values at a dosage of $1.5 \mathrm{~g} / \mathrm{kg} /$ day. Each of the three high dosages $(1.0 \sim 1.5 \mathrm{~g} / \mathrm{kg} /$ day $)$ maintained AA blood levels at $10-20 \mathrm{mmol} / \mathrm{L}$ for approximately $3 \mathrm{~h}$, and there were no significant differences (two-tailed Student's $t$ test) in the $C_{\max }$ values between day 1 and day 3 of administration for each dose. Although this effective level $(10-20 \mathrm{mmol} / \mathrm{L})$ of AA was similar between the three high dosages $(1.0 \sim 1.5 \mathrm{~g} / \mathrm{kg} /$ day), fixed-rate infusion trials were carried out.

When $1.5 \mathrm{~g} / \mathrm{kg}$ of vitamin $\mathrm{C}$ was given during fixed-rate infusion $(0.6,0.8,1 \mathrm{~g} / \mathrm{min})$, plasma concentrations were much higher than when the vitamin was given at a fixed infusion time (Additional file 2: Figure S2). The mean peak values from the fixed-rate administration were 25.58 $\mathrm{mmol} / \mathrm{L}$, at $10-20 \mathrm{mmol} / \mathrm{L}$ for approximately $3 \mathrm{~h}$. As 
Table 2 Treatment-emergent adverse events for the study cohort $(N=36)$

\begin{tabular}{|c|c|c|c|c|c|}
\hline \multirow[t]{2}{*}{ Adverse event } & \multicolumn{4}{|l|}{ No (\%) } & \multirow[b]{2}{*}{ Grade4 } \\
\hline & All grades & Grade 1 & Grade 2 & $\overline{\text { Grade } 3}$ & \\
\hline Peripheral sensory neuropathy & $18(50.0)$ & $9(25.0)$ & $8(22.2)$ & $1(2.8)$ & $0(0)$ \\
\hline Nausea & $14(38.9)$ & $13(36.1)$ & $1(2.8)$ & $0(0)$ & $0(0)$ \\
\hline Vomiting & $13(36.1)$ & $8(22.2)$ & $4(11.1)$ & $1(2.8)$ & $0(0)$ \\
\hline Neutropenia & $10(27.8)$ & $0(0)$ & $4(11.1)$ & $5(13.9)$ & $1(2.8)$ \\
\hline Abdominal pain & $9(25.0)$ & $9(25.0)$ & $0(0)$ & $0(0)$ & $0(0)$ \\
\hline Decreased appetite & $8(22.2)$ & $8(22.2)$ & $0(0)$ & $0(0)$ & $0(0)$ \\
\hline leukopenia & $8(22.2)$ & $3(8.2)$ & $4(11.1)$ & $1(2.8)$ & $0(0)$ \\
\hline Diarrhea & $7(19.4)$ & $5(13.9)$ & $1(2.8)$ & $1(2.8)$ & $0(0)$ \\
\hline Alopecia & $4(11.1)$ & $4(11.1)$ & $0(0)$ & $0(0)$ & $0(0)$ \\
\hline Malaise & $4(11.1)$ & $4(11.1)$ & $0(0)$ & $0(0)$ & $0(0)$ \\
\hline Fatigue & $3(8.2)$ & $3(8.2)$ & $0(0)$ & $0(0)$ & $0(0)$ \\
\hline Hand-foot syndrome & $3(8.2)$ & $3(8.2)$ & $0(0)$ & $0(0)$ & $0(0)$ \\
\hline Mucositis oral & $3(8.2)$ & $3(8.2)$ & $0(0)$ & $0(0)$ & $0(0)$ \\
\hline Hemorrhage, vagina & $2(5.6)$ & $2(5.6)$ & $0(0)$ & $0(0)$ & $0(0)$ \\
\hline Dyspnea & $2(5.6)$ & $2(5.6)$ & $0(0)$ & $0(0)$ & $0(0)$ \\
\hline Thrombocytopenia & $2(5.6)$ & $1(2.8)$ & $1(2.8)$ & $0(0)$ & $0(0)$ \\
\hline Anemia & $2(5.6)$ & $1(2.8)$ & $1(2.8)$ & $0(0)$ & $0(0)$ \\
\hline Hyperpigmentation & $2(5.6)$ & $2(5.6)$ & $0(0)$ & $0(0)$ & $0(0)$ \\
\hline Dizziness & $2(5.6)$ & $2(5.6)$ & $0(0)$ & $0(0)$ & $0(0)$ \\
\hline Pain & $2(5.6)$ & $2(5.6)$ & $0(0)$ & $0(0)$ & $0(0)$ \\
\hline Gingival pain & $2(5.6)$ & $2(5.6)$ & $0(0)$ & $0(0)$ & $0(0)$ \\
\hline Fever & $2(5.6)$ & $2(5.6)$ & $0(0)$ & $0(0)$ & $0(0)$ \\
\hline Palpitations & $2(5.6)$ & $2(5.6)$ & $0(0)$ & $0(0)$ & $0(0)$ \\
\hline Upper respiratory tract infection & $1(2.8)$ & $1(2.8)$ & $0(0)$ & $0(0)$ & $0(0)$ \\
\hline Skin rash & $1(2.8)$ & $0(0)$ & $1(2.8)$ & $0(0)$ & $0(0)$ \\
\hline Hematuresis & $1(2.8)$ & $1(2.8)$ & $0(0)$ & $0(0)$ & $0(0)$ \\
\hline Weight loss & $1(2.8)$ & $1(2.8)$ & $0(0)$ & $0(0)$ & $0(0)$ \\
\hline Tinnitus & $1(2.8)$ & $1(2.8)$ & $0(0)$ & $0(0)$ & $0(0)$ \\
\hline Cough & $1(2.8)$ & $1(2.8)$ & $0(0)$ & $0(0)$ & $0(0)$ \\
\hline Epistaxis & $1(2.8)$ & $1(2.8)$ & $0(0)$ & $0(0)$ & $0(0)$ \\
\hline Productive cough & $1(2.8)$ & $1(2.8)$ & $0(0)$ & $0(0)$ & $0(0)$ \\
\hline Hypertension & $1(2.8)$ & $1(2.8)$ & $0(0)$ & $0(0)$ & $0(0)$ \\
\hline
\end{tabular}

shown in Additional file 3: Table S1, the volumes of distribution and clearance were similar to those parameters in the fixed infusion time group. When the recommended subsequent dose was administered, the plasma concentrations of AA were maintained at $10-20 \mathrm{mmol} / \mathrm{L}$ for more than $4 \mathrm{~h}$.

\section{Exploratory efficacy findings}

Of the 36 patients enrolled, 24 (23 with mCRC and 1 with mGC) were evaluated for tumor response. The best overall responses included a partial response in fourteen patients (objective response rate, 58.3\%) and stable disease in nine (37.5\%), giving a disease control rate of $95.8 \%$. Among 22 patients with $\mathrm{mCRC}$ receiving first-line therapy, the objective response rate was $59.1 \%$ and the disease control rate was $95.5 \%$. Figure 3 shows the maximum percentage change from the baseline in measurable target lesions. Notably, patients with wild-type RAS/BRAF or mutant KRAS or BRAF all showed good response to the treatment. No difference in efficacy was found between mCRC patients with wild-type RAS/BRAF and mutant KRAS or BRAF according to Fisher's test ( $p=0.387)$. 
A

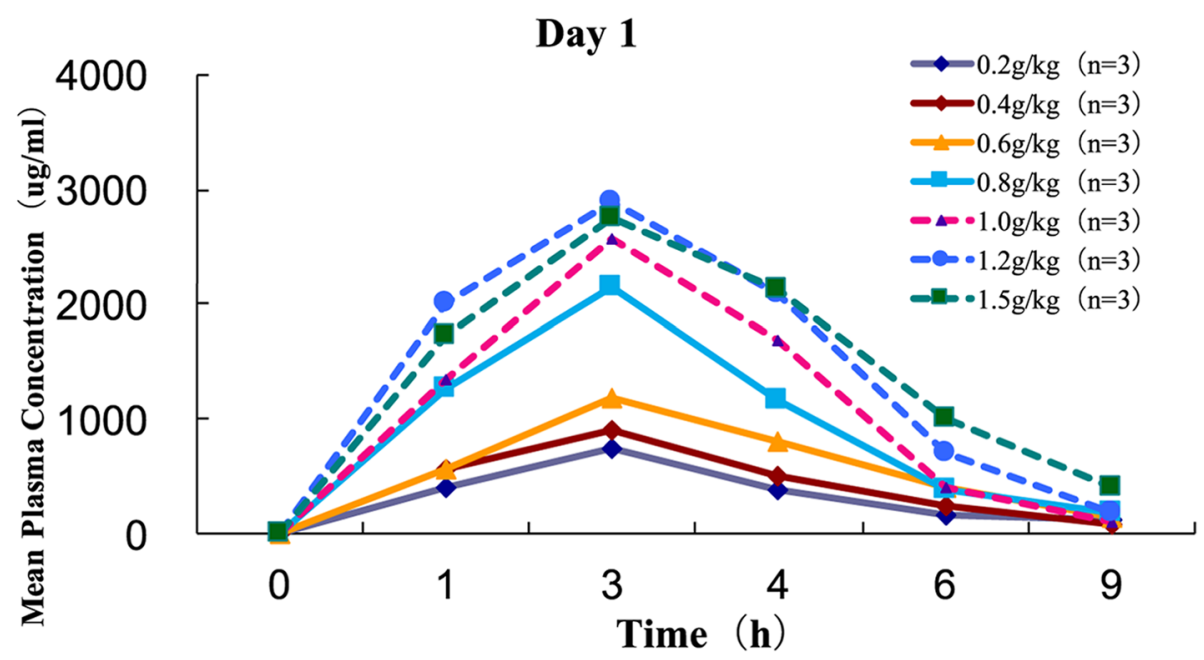

B

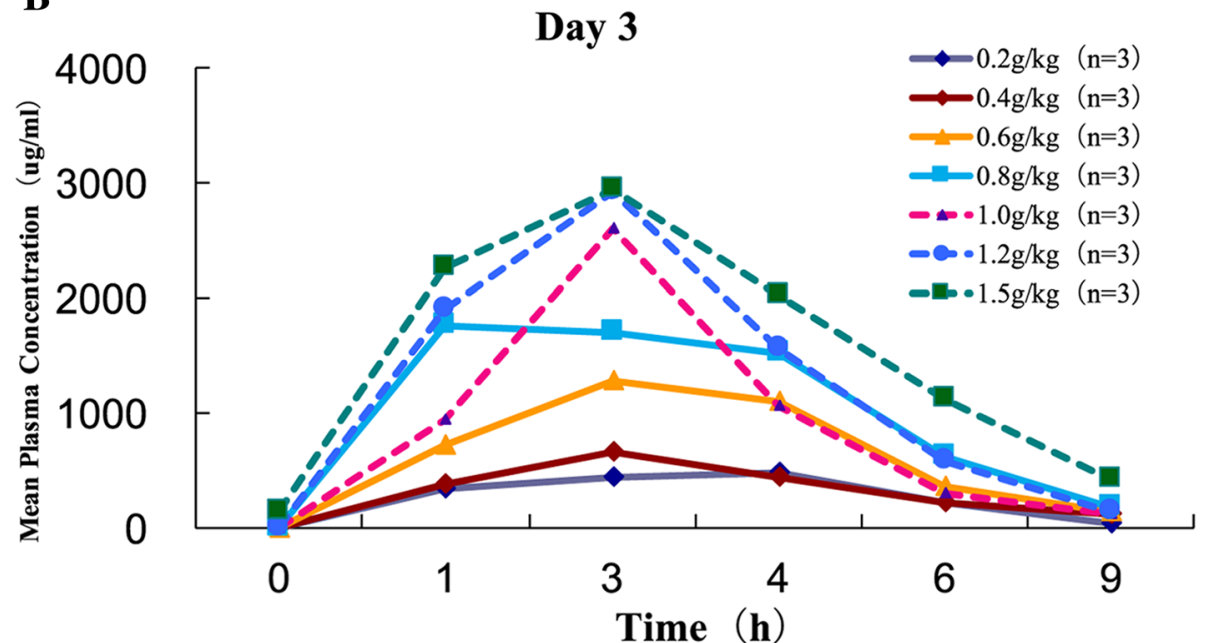

Fig. 2 Mean plasma concentration-time curve of ascorbic acid after $0.2 \sim 1.5 \mathrm{~g} / \mathrm{kg}$ administration to cancer patients $(n=3)$ on Day 1 (a) and Day 3 (b). After i.v. administration, the plasma concentrations of ascorbic acid rose gradually and peaked at $3 \mathrm{~h}$. The Cmax, and AUC values of ascorbic acid displayed dose-dependent increases. Ascorbic acid concentrations in the high-dose groups remained at 10-20 mmol/L for approximately $3 \mathrm{~h}$ and showed no accumulation in the body during 3 daily administrations. Curves varying in linetypes and colors correspond to different administration dosages of ascorbic acid

With a median follow-up of 8.6 months (interquartile range, 7.4-11.4) at the cutoff date for the collection of survival data (May 15, 2018), 17 PFS events (16 disease progression and 1 death) were recorded. The median PFS for the entire cohort was 8.8 months. Of note, the PFS of the two patients with mutant BRAF was 9.6 and 5.1 months.

\section{Discussion}

To the best of our knowledge, this is the first study investigating the safety profile and MTD for the combination of AA and chemotherapy among the East Asian population with advanced solid tumors. Our findings suggest that AA at $1.5 \mathrm{~g} / \mathrm{kg}$ once daily for three consecutive days can be safely coadministered with mFOLFOX6 or FOLFIRI in a 14-day cycle, and although preliminary, this combination exhibited encouraging clinical efficacy.

The most commonly reported side effects were attributable to high-dose AA and included headache, light-headedness, dry mouth, and gastrointestinal toxicity due to rapid infusion and high osmotic load [19-21]. However, these adverse events were generally uncommon in this study. Grade 1-2 gastrointestinal toxicities with incidences of $20-40 \%$ were recorded, but their relevance to chemotherapy could not be excluded.

Previous studies have suggested that the combination of AA with chemotherapeutic agents reduced chemotherapy- 
Table 3 Pharmacokinetic values

\begin{tabular}{|c|c|c|c|c|c|c|c|c|}
\hline & Unit & $0.2 \mathrm{~g} / \mathrm{kg}(n=3)$ & $0.4 \mathrm{~g} / \mathrm{kg}(n=3)$ & $0.6 \mathrm{~g} / \mathrm{kg}(n=3)$ & $0.8 \mathrm{~g} / \mathrm{kg}(n=3)$ & $1 \mathrm{~g} / \mathrm{kg}(n=2)$ & $1.2 \mathrm{~g} / \mathrm{kg}(n=3)$ & $1.5 \mathrm{~g} / \mathrm{kg}(n=3)$ \\
\hline \multirow[t]{5}{*}{$\overline{D 1}$} & $\mathrm{Cmax}(\mathrm{mmol} / \mathrm{l})$ & $4.2 \pm 3.9$ & $5.1 \pm 1.1$ & $7.4 \pm 1.2$ & $12.1 \pm 5.7$ & $14.0 \pm 3.9$ & $16.4 \pm 2.5$ & $17.4 \pm 7.2$ \\
\hline & $\mathrm{t} 1 / 2(\mathrm{~h})$ & $3.1 \pm 1.2$ & $1.8 \pm 0.6$ & $2.0 \pm 0.2$ & $1.9 \pm 0.05$ & $1.5 \pm 0.2$ & $1.4 \pm 0.09$ & $1.97 \pm 0.8$ \\
\hline & $\operatorname{AUC}\left(\mathrm{mmol} / \mathrm{I}^{*} \mathrm{~h}\right)$ & $21.0 \pm 18.0$ & $22.5 \pm 8.8$ & $31.5 \pm 3.0$ & $49.0 \pm 15.8$ & $55.3 \pm 2.3$ & $73.3 \pm 12.3$ & $82.3 \pm 43.3$ \\
\hline & $\mathrm{Vz}(\mathrm{ml})$ & $15.3 \pm 5.6$ & $13.6 \pm 1.7$ & $14.2 \pm 3.8$ & $14.2 \pm 2.4$ & $12.7 \pm 1.6$ & $11.5 \pm 1.3$ & $14.9 \pm 4.0$ \\
\hline & $\mathrm{Cl}(\mathrm{ml} / \mathrm{h})$ & $4.1 \pm 2.5$ & $5.8 \pm 2.9$ & $4.9 \pm 1.4$ & $5.1 \pm 1.0$ & $6.0 \pm 0.03$ & $5.5 \pm 0.4$ & $5.5 \pm 1.7$ \\
\hline \multirow[t]{5}{*}{ D3 } & $\mathrm{Cmax}(\mathrm{mmol} / \mathrm{l})$ & $3.5 \pm 1.7$ & $3.7 \pm 0.9$ & $7.2 \pm 1.1$ & $11.2 \pm 3.3$ & $9.5 \pm 3.0$ & $16.5 \pm 1.5$ & $17.8 \pm 5.5$ \\
\hline & $\mathrm{t} 1 / 2(\mathrm{~h})$ & $1.9 \pm 0.8$ & $2.4 \pm 0.9$ & $1.7 \pm 0.4$ & $1.6 \pm 0.1$ & $1.5 \pm 0.2$ & $1.5 \pm 0.06$ & $1.98 \pm 0.6$ \\
\hline & $\mathrm{AUC}(\mathrm{mmol} / \mathrm{I}$ *h) & $14.9 \pm 7.4$ & $19.0 \pm 7.5$ & $34.1 \pm 3.9$ & $54.8 \pm 16.1$ & $35.4 \pm 15.8$ & $65.5 \pm 8.0$ & $89.5 \pm 52.4$ \\
\hline & $\mathrm{Vz}(\mathrm{ml})$ & $12.2 \pm 7.6$ & $21.4 \pm 3.2$ & $10.6 \pm 3.6$ & $10.9 \pm 2.7$ & $22.6 \pm 7.7$ & $13.3 \pm 3.4$ & $14.2 \pm 3.6$ \\
\hline & $\mathrm{Cl}(\mathrm{ml} / \mathrm{h})$ & $4.4 \pm 1.8$ & $6.9 \pm 3.5$ & $4.4 \pm 0.6$ & $4.6 \pm 1.0$ & $10.5 \pm 5.0$ & $6.2 \pm 1.4$ & $5.3 \pm 2.1$ \\
\hline
\end{tabular}

associated toxicity $[18,22]$. In a pilot phase $1 / 2$ a trial for stage III/IV ovarian cancer, the addition of AA to paclitaxel and carboplatin substantially decreased diverse categories of toxicity, including neurologic, dermatologic, and bone marrow toxicity, and toxicities in the renal/genitourinary and gastrointestinal systems [18]. Additionally, AA did not appear to increase the toxicity of gemcitabine, arsenic trioxide, melphalan, bortezomib, or dexamethasone [19, 23, 24]. In line with these findings, the current study showed markedly decreased all-grade and grade $\geq 3$ bone marrow and gastrointestinal toxic effects compared with previous trials investigating the same chemotherapeutic regimens in $\mathrm{mCRC}$ or $\mathrm{mGC}$. For instance, the incidence of grade $\geq 3$ neutropenia was $13.9 \%$ in the current study, in contrast to approximately $30 \%$ in previous studies. [25, 26] Moreover, neurotoxicity in patients treated with at least 6 cycles of oxaliplatin was much less common than that in trials discontinuing oxaliplatin after six cycles (grade 3 neurotoxicity, $2.8 \%$ in our study versus approximately $20 \%$ in prior studies) [27, 28].

Intravenous AA administered at a dosage of $1.5 \mathrm{~g} / \mathrm{kg}$ three times weekly appears to be safe and free of important toxicity in appropriately screened patients with advanced untreatable malignancies. A phase 1 trial with a fixed infusion time stopped the dose escalation at $1.5 \mathrm{~g} /$ $\mathrm{kg}$ when peak blood levels approached a plateau of 10

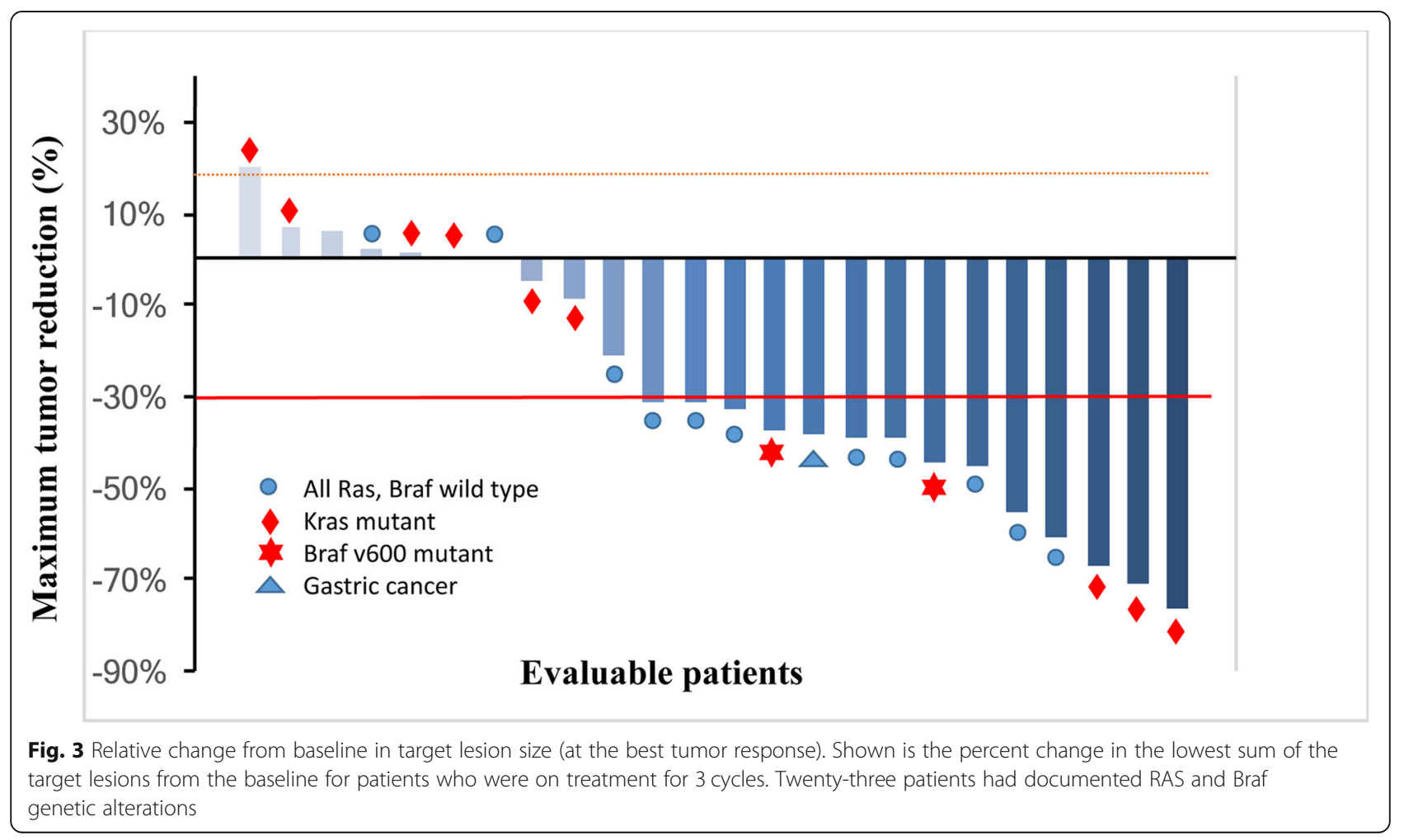


$20 \mathrm{mmol} / \mathrm{L}$, the level that can inhibit tumor growth in mice. Although the Cmax and AUC values for AA increased proportionately with AA doses between 0.2 and $1.5 \mathrm{~g} / \mathrm{kg}$, the values of these parameters did not increase much further at doses higher than $1.5 \mathrm{~g} / \mathrm{kg}$. Fixed-rate infusions at $0.6 \mathrm{~g} / \mathrm{min}$ produced similar Cmax and AUC values with a 3 -h infusion, and fixed-rate infusions at $0.8 \mathrm{~g} / \mathrm{min}$ and $1 \mathrm{~g} / \mathrm{min}$ resulted in slightly higher Cmax and AUC values than with the 3 -h infusion. However, two patients complained about palpitation during the AA infusion due to the high-speed infusion rate. Therefore, a 3-h infusion is recommended for future studies.

A previous study has found that mutant KRAS or BRAF CRC cells exhibit high expression of GLUT1, leading to increased uptake of the oxidized form of vitamin C, dehydroascorbate (DHA). This increased DHA uptake causes oxidative stress as intracellular DHA is reduced to vitamin $\mathrm{C}$, depleting glutathione and leading to energetic crisis and cell death. However, this phenomenon was only observed in KRAS and BRAF mutant cancer cells. No difference in efficacy was found between mCRC patients with wild-type RAS and mutant RAS or BRAF in our study. Therefore, not only patients with mutant RAS or BRAF but also those with wild-type RAS and BRAF should be tested in future studies. Of note, two patients with mutant BRAF responded well to treatment.

In preclinical studies, synergy has been demonstrated between AA and chemotherapy agents such as oxaliplatin [17]. Our group found that the combination of AA with chemotherapeutic agents, including oxaliplatin and irinotecan, achieved synergistic inhibitory effects in gastric cancer PDX models via an increase in oxidative stress [11]. Considering such synergistic effects, we modified the administration schedule of AA from a previous study [17], and AA was given once daily for three consecutive days in a 14-day cycle alongside mFOLFOX6 or FOLFIRI. Preclinical evidence suggests that apoptotic cell death occurs in many cancer cell lines exposed to AA concentrations $>5 \mathrm{mmol} / \mathrm{L}$ for $<1 \mathrm{~h}$ [16]. Comparable to that with AA monotherapy at $1.5 \mathrm{~g} / \mathrm{kg}$ in the Caucasian population [17], our PK analysis revealed that $\mathrm{AA}$ at $1.5 \mathrm{~g} / \mathrm{kg}$ sustained a mean plasma AA concentration exceeding $5 \mathrm{mmol} / \mathrm{L}$ for at least $5 \mathrm{~h}$. Even though only half of the patients with $\mathrm{mCRC}$ received concomitant bevacizumab in this study, an objective response rate of $59.1 \%$ was achieved with the addition of AA to FOLFOX \pm bevacizumab as first-line therapy for mCRC, which compared favorably with that reported in previous trials of first-line FOLFOX plus bevacizumab. [25, 26] Considering its promising effect in $\mathrm{mCRC}$ and $\mathrm{mGC}$, the additional efficacy of treatment when AA is combined with chemotherapy is worth investigating in larger clinical trials.

\section{Conclusions}

In summary, the combination of AA at $1.5 \mathrm{~g} / \mathrm{kg}$ once daily for three consecutive days with mFOLFOX6 or FOLFIRI with or without bevacizumab every 14 days exhibits a favorable safety profile and potential clinical efficacy in patients with mCRC or mGC. As a result, a randomized phase III study is ongoing investigating the additional efficacy of treatment when AA is combined with mFOLFOX6 \pm bevacizumab as first-line therapy for patients with mCRC (NCT02969681).

\section{Additional files}

Additional file 1: Figure S1. Evaluation of the ascorbic acid dose proportionality in part 1 of the study. The mean AA Cmax and AUC are presented vs increasing doses of AA (measured after the first administered dose of AA) on day 1 and day 3. AUC 0-9: area under the plasma concentration-time curve from time zero to hour 9; Cmax: maximum plasma concentration. (PDF $21 \mathrm{~kb}$ )

Additional file 2: Figure S2. Mean plasma concentration-time curve of ascorbic acid after fixed-rate infusions at $0.6,0.8,1.0 \mathrm{~g} / \mathrm{min}$ to cancer patients. After i.v. administration, the plasma concentrations of ascorbic acid rose gradually and peaked at $3 \mathrm{~h}$. Cmax, and AUC values of ascorbic acid display dose-dependent increases. Ascorbic acid concentrations in the high-dose groups remained at 10-20 mmol/L for more than $4 \mathrm{~h}$ and showed no accumulation in the body during the administrations. (PDF $20 \mathrm{~kb}$ )

Additional file 3: Table S1. Pharmacokinetic values. The volumes of distribution and clearance were similar to those parameters in the fixed infusion time group. When the recommended subsequent dose was administered, the plasma concentrations of AA were maintained at $10-20 \mathrm{mmol} / \mathrm{L}$ for more than $4 \mathrm{~h}$. (DOCX $17 \mathrm{~kb}$ )

\section{Abbreviations}

AA: Ascorbic acid; AUC: Area under the curve; CTCAE: Common terminology criteria for adverse events; DHA: Dehydroascorbate; DLT: Dose-limiting toxicity; ECOG: Eastern cooperative oncology group; mCRC: Metastatic colorectal cancer; mGC: Metastatic GC; MTD: Maximum tolerated dose; PDX: Patient-derived xenograft; PFS: Progression-free survival; PK: Pharmacokinetic; RECIST: Response Evaluation Criteria in Solid Tumors; RP2D: Recommended phase 2 dose; ULN: Upper limit of normal

\section{Acknowledgements}

We are grateful to all the patients who participated in this trial and their families.

\section{Funding}

This work was supported, in part, by the National Key Research and Development Program of China (2017YFC1308900), the National Natural Science Foundation of China (81872011), the Natural Science Foundation of Guangdong Province (2014A030312015), Science and Technology Program of Guangdong (2015B020232008), grants from the Science and Technology Program of Guangzhou (15570006), the Pearl River Nova Program of Guangzhou (201610010068) and the Fundamental Research Funds for the Central Universities (14ykpy40). Feng Wang is a recipient of the Outstanding Young Talents Program of Sun Yat-sen University and the Young Physician Scientist Program of Sun Yat-sen University Cancer Center. The funders of this study had no role in its design, data collection, data analysis, data interpretation, or writing of the report.

\section{Availability of data and materials}

The datasets used and/or analyzed during the current study are available from the corresponding author on reasonable request.

\section{Authors' contributions}

All authors were involved in manuscript writing. $\mathrm{RHX}$ and $\mathrm{FW}$ contributed to the conception and design of the study. FW, MMH, ZXW, YJ, CR, SMS, SZC, 
ZDL, JJH, ZQW, FHW, DSW and YHL contributed to the collection and assembly of data. All authors contributed to the data analysis and interpretation. RHX, FW and MMH had access to the raw data. ZXW, SL and BTB created the tables and figures. All authors had the opportunity to review the plan and outcome of analysis, participated in the preparation of this article, and provided final approval.

\section{Ethics approval and consent to participate}

The trial was approved by the Independent Institute Research Ethics Committee at the Sun Yat-sen University Cancer Center prior to initiation. This trial was conducted in accordance with the Declaration of Helsinki, the guidelines for Good Clinical Practice, the European Union Clinical Trial Directive, and local regulations. All participants provided written informed consent.

\section{Consent for publication}

Not applicable.

\section{Competing interests}

The authors declare that they have no potential conflicts of interest to disclose.

\section{Publisher's Note}

Springer Nature remains neutral with regard to jurisdictional claims in published maps and institutional affiliations.

\section{Author details}

${ }^{1}$ State Key Laboratory of Oncology in South China, Collaborative Innovation Center for Cancer Medicine, Sun Yat-sen University Cancer Center, Guangzhou 510060, People's Republic of China. 'Department of Medical Oncology, Sun Yat-sen University Cancer Center, Guangzhou 510060, China ${ }^{3}$ Department of Clinical Trial Center, Sun Yat-sen University Cancer Center, Guangzhou 510060, China.

Received: 15 November 2018 Accepted: 8 May 2019

Published online: 16 May 2019

\section{References}

1. Ohno S, Ohno Y, Suzuki N, Soma G, Inoue M. High-dose vitamin C (ascorbic acid) therapy in the treatment of patients with advanced cancer. Anticancer Res. 2009;29(3):809-15.

2. Block G. Epidemiologic evidence regarding vitamin C and cancer. Am J Clin Nutr. 1991;54(6 Suppl):1310S-4S.

3. Lin J, Cook NR, Albert C, Zaharris E, Gaziano JM, Van Denburgh M, Buring JE, Manson JE. Vitamins $C$ and $E$ and beta carotene supplementation and cancer risk: a randomized controlled trial. J Natl Cancer Inst. 2009;101(1):14-23.

4. Gaziano JM, Glynn RJ, Christen WG, Kurth T, Belanger C, MacFadyen J, Bubes $V$, Manson JE, Sesso HD, Buring JE. Vitamins $E$ and $C$ in the prevention of prostate and total cancer in men: the Physicians' health study II randomized controlled trial. JAMA. 2009;301(1):52-62.

5. Cameron E, Pauling L. Supplemental ascorbate in the supportive treatment of cancer: prolongation of survival times in terminal human cancer. Proc Natl Acad Sci U S A. 1976;73(10):3685-9.

6. Cameron E, Pauling L. Supplemental ascorbate in the supportive treatment of cancer: reevaluation of prolongation of survival times in terminal human cancer. Proc Natl Acad Sci U S A. 1978;75(9):4538-42.

7. Creagan ET, Moertel CG, O'Fallon JR, Schutt AJ, O'Connell MJ, Rubin J, Frytak S. Failure of high-dose vitamin C (ascorbic acid) therapy to benefit patients with advanced cancer. A controlled trial. N Engl J Med. 1979;301(13):687-90.

8. Moertel CG, Fleming TR, Creagan ET, Rubin J, O'Connell MJ, Ames MM High-dose vitamin $C$ versus placebo in the treatment of patients with advanced cancer who have had no prior chemotherapy. A randomized double-blind comparison. N Engl J Med. 1985;312(3):137-41.

9. Du J, Cullen JJ, Buettner GR. Ascorbic acid: chemistry, biology and the treatment of cancer. Biochim Biophys Acta. 2012;1826(2):443-57.

10. Schoenfeld JD, Sibenaller ZA, Mapuskar KA, Wagner BA, Cramer-Morales KL, Furqan M, Sandhu S, Carlisle TL, Smith MC, Abu Hejleh T, et al. O2(-) and $\mathrm{H} 2 \mathrm{O} 2$-mediated disruption of Fe metabolism causes the differential susceptibility of NSCLC and GBM Cancer cells to pharmacological ascorbate. Cancer Cell. 2017:31(4):487-500 e488.

11. Lu YX, Wu QN, Chen DL, Chen LZ, Wang ZX, Ren C, Mo HY, Chen Y, Sheng $\mathrm{H}$, Wang $\mathrm{YN}$, et al. Pharmacological ascorbate suppresses growth of gastric
Cancer cells with GLUT1 overexpression and enhances the efficacy of Oxaliplatin through redox modulation. Theranostics. 2018:8(5):1312-26.

12. Yun J, Mullarky E, Lu C, Bosch KN, Kavalier A, Rivera K, Roper J, Chio II, Giannopoulou EG, Rago C, et al. Vitamin C selectively kills KRAS and BRAF mutant colorectal cancer cells by targeting GAPDH. Science. 2015;350(6266): 1391-6.

13. Cimmino L, Dolgalev I, Wang Y, Yoshimi A, Martin GH, Wang J, Ng V, Xia B, Witkowski MT, Mitchell-Flack M, et al. Restoration of TET2 function blocks aberrant self-renewal and leukemia progression. Cell. 2017;170(6):1079-1095.e1020.

14. Bram S, Froussard P, Guichard M, Jasmin C, Augery Y, Sinoussi-Barre F, Wray W. Vitamin C preferential toxicity for malignant melanoma cells. Nature. 1980;284(5757):629-31

15. Sestili P, Brandi G, Brambilla L, Cattabeni F, Cantoni O. Hydrogen peroxide mediates the killing of U937 tumor cells elicited by pharmacologically attainable concentrations of ascorbic acid: cell death prevention by extracellular catalase or catalase from cocultured erythrocytes or fibroblasts. J Pharmacol Exp Ther. 1996;277(3):1719-25.

16. Chen Q, Espey MG, Krishna MC, Mitchell JB, Corpe CP, Buettner GR, Shacter E, Levine M. Pharmacologic ascorbic acid concentrations selectively kill cancer cells: action as a pro-drug to deliver hydrogen peroxide to tissues. Proc Natl Acad Sci U S A. 2005;102(38):13604-9.

17. Hoffer $\amalg$, Levine M, Assouline S, Melnychuk D, Padayatty SJ, Rosadiuk K Rousseau C, Robitaille L, Miller WH Jr. Phase I clinical trial of i.v. ascorbic acid in advanced malignancy. Ann Oncol. 2008;19(11):1969-74.

18. Ma Y, Chapman J, Levine M, Polireddy K, Drisko J, Chen Q. High-dose parenteral ascorbate enhanced chemosensitivity of ovarian cancer and reduced toxicity of chemotherapy. Sci Transl Med. 2014;6(222):222ra218.

19. Welsh JL, Wagner BA, van't Erve TJ, Zehr PS, Berg DJ, Halfdanarson TR, Yee NS, Bodeker KL, Du J, Roberts LJ 2nd, et al. Pharmacological ascorbate with gemcitabine for the control of metastatic and node-positive pancreatic cancer (PACMAN): results from a phase I clinical trial. Cancer Chemother Pharmacol. 2013;71(3):765-75.

20. Stephenson CM, Levin RD, Spector T, Lis CG. Phase I clinical trial to evaluate the safety, tolerability, and pharmacokinetics of high-dose intravenous ascorbic acid in patients with advanced cancer. Cancer Chemother Pharmacol. 2013;72(1):139-46.

21. Riordan HD, Casciari JJ, Gonzalez MJ, Riordan NH, Miranda-Massari JR, Taylor $P$, Jackson JA. A pilot clinical study of continuous intravenous ascorbate in terminal cancer patients. P R Health Sci J. 2005;24(4):269-76.

22. Vollbracht C, Schneider B, Leendert V, Weiss G, Auerbach L, Beuth J. Intravenous vitamin $C$ administration improves quality of life in breast cancer patients during chemo-/radiotherapy and aftercare: results of a retrospective, multicentre, epidemiological cohort study in Germany. In Vivo. 2011;25(6):983-90.

23. Berenson JR, Matous J, Swift RA, Mapes R, Morrison B, Yeh HS. A phase I/II study of arsenic trioxide/bortezomib/ascorbic acid combination therapy for the treatment of relapsed or refractory multiple myeloma. Clin Cancer Res. 2007:13(6):1762-8

24. Abou-Jawde RM, Reed J, Kelly M, Walker E, Andresen S, Baz R, Karam MA, Hussein M. Efficacy and safety results with the combination therapy of arsenic trioxide, dexamethasone, and ascorbic acid in multiple myeloma patients: a phase 2 trial. Med Oncol. 2006;23(2):263-72.

25. Venook AP, Niedzwiecki D, Lenz HJ, Innocenti F, Fruth B, Meyerhardt JA, Schrag D, Greene C, O'Neil BH, Atkins JN, et al. Effect of first-line chemotherapy combined with Cetuximab or bevacizumab on overall survival in patients with KRAS wild-type advanced or metastatic colorectal Cancer: a randomized clinical trial. JAMA. 2017;317(23):2392-401.

26. Yamada Y, Takahari D, Matsumoto H, Baba H, Nakamura M, Yoshida K, Yoshida M, Iwamoto S, Shimada K, Komatsu Y, et al. Leucovorin, fluorouracil, and oxaliplatin plus bevacizumab versus S-1 and oxaliplatin plus bevacizumab in patients with metastatic colorectal cancer (SOFT): an open-label, non-inferiority, randomised phase 3 trial. Lancet Oncol. 2013;14(13):1278-86.

27. Tournigand C, Cervantes A, Figer A, Lledo G, Flesch M, Buyse M, Mineur L, Carola E, Etienne PL, Rivera F, et al. OPTIMOX1: a randomized study of FOLFOX4 or FOLFOX7 with oxaliplatin in a stop-and-go fashion in advanced colorectal cancer--a GERCOR study. J Clin Oncol. 2006;24(3):394-400.

28. Diaz-Rubio E, Gomez-Espana A, Massuti B, Sastre J, Abad A, Valladares M, Rivera F, Safont MJ, Martinez de Prado P, Gallen M, et al. First-line XELOX plus bevacizumab followed by XELOX plus bevacizumab or single-agent bevacizumab as maintenance therapy in patients with metastatic colorectal cancer: the phase III MACRO TTD study. Oncologist. 2012;17(1):15-25. 